\title{
Existence of common fixed points using Bregman nonexpansive retracts and Bregman functions in Banach spaces
}

\author{
Nawab Hussain ${ }^{1 *}$, Eskandar Naraghirad ${ }^{1,2}$ and Abdullah Alotaibi ${ }^{1}$
}

${ }^{*}$ Correspondence:

nhusain@kau.edu.sa

${ }^{1}$ Department of Mathematics, King

Abdulaziz University, P.O. Box 80203

Jeddah, 21589, Saudi Arabia

Full list of author information is

available at the end of the article

\begin{abstract}
In this paper, we first introduce the concepts of Bregman nonexpansive retract and Bregman one-local retract and then use these concepts to establish the existence of common fixed points for Banach operator pairs in the framework of reflexive Banach spaces. No compactness assumption is imposed either on $C$ or on $T$, where $C$ is a closed and convex subset of a reflexive Banach space $E$ and $T: C \rightarrow C$ is a Bregman nonexpansive mapping. We also establish the well-known De Marr theorem for a Banach operator family of Bregman nonexpansive mappings.

MSC: Primary 06F30; 46B20; 47E10

Keywords: reflexive Banach space; Gâteaux differentiable function; Bregman projection; Bregman distance; Bregman nonexpansive mapping; Banach operator pair
\end{abstract}

\section{Introduction}

This paper is motivated by the recent papers [1-4]. In [3] the authors study different questions related to common fixed points of Banach operator pairs in hyperconvex spaces. In [2] the authors introduced the concept of NR-maps and then they used this concept to establish the existence of common fixed points for Banach operator pairs in the context of uniformly convex geodesic metric spaces. In our present work, using Bregman functions, we propose to consider similar questions on reflexive Banach spaces under the mildest weaker conditions we may impose. More precisely, we first introduce the concepts of Bregman NR-map and Bregman one-local retract and then use these concepts to establish the existence of common fixed points for Banach operator pairs in reflexive Banach spaces. No compactness assumption is imposed either on $C$ or on $T$, where $C$ is a closed and convex subset of a reflexive Banach space $E$ and $T: C \rightarrow C$ is a Bregman nonexpansive mapping. For a recent survey on the existence of fixed points in geodesic spaces, we refer the readers to $[1,5]$.

The celebrated result on the existence of a common fixed point for a nonexpansive commutative family was first established by De Marr [6] under the assumption that $C$ is a compact convex subset of a normed space $X$. In 1965, Browder [7] obtained the corresponding result under the assumption that $C$ is a bounded, closed and convex subset of a uniformly convex Banach space $X$. In 1992, Khamsi et al. [8] established the above mentioned results for a finite as well as an arbitrary commutative family of maps in hyperconvex metric spaces. Recently, Espìnola and Hussain [9] proved De Marr's theorem in uniformly con-

c) 2013 Hussain et al.; licensee Springer. This is an Open Access article distributed under the terms of the Creative Commons Attribution License (http://creativecommons.org/licenses/by/2.0), which permits unrestricted use, distribution, and reproduction in any medium, provided the original work is properly cited. 
vex metric spaces of type (T). More recently, Hussain et al. [3] extended De Marr's result to the family of symmetric Banach operator pairs in hyperconvex metric spaces (see also [10-12]).

Throughout this paper, we denote the set of real numbers and the set of positive integers by $\mathbb{R}$ and $\mathbb{N}$, respectively. Let $E$ be a real Banach space and let $C$ be a nonempty subset of $E$. Let $T: C \rightarrow E$ be a mapping. We denote by $F(T)$ the set of fixed points of $T$, i.e., $F(T)=\{x \in C: T x=x\}$.

Let $E$ be a Banach space with the norm $\|\cdot\|$ and the dual space $E^{*}$. For any $x \in E$, we denote the value of $x^{*} \in E^{*}$ at $x$ by $\left\langle x, x^{*}\right\rangle$. When $\left\{x_{n}\right\}_{n \in \mathbb{N}}$ is a sequence in $E$, we denote the strong convergence of $\left\{x_{n}\right\}_{n \in \mathbb{N}}$ to $x \in E$ by $x_{n} \rightarrow x$ and the weak convergence by $x_{n} \rightarrow x$. The modulus $\delta$ of the convexity of $E$ is denoted by

$$
\delta(\epsilon)=\inf \left\{1-\frac{\|x+y\|}{2}:\|x\| \leq 1,\|y\| \leq 1,\|x-y\| \geq \epsilon\right\}
$$

for every $\epsilon$ with $0 \leq \epsilon \leq 2$. A Banach space $E$ is said to be uniformly convex if $\delta(\epsilon)>0$ for every $\epsilon>0$. Let $S_{E}=\{x \in E:\|x\|=1\}$. The norm of $E$ is said to be Gâteaux differentiable if for each $x, y \in S_{E}$, the limit

$$
\lim _{t \rightarrow 0} \frac{\|x+t y\|-\|x\|}{t}
$$

exists. In this case, $E$ is called smooth. If the limit (1.1) is attained uniformly in $x, y \in S_{E}$, then $E$ is called uniformly smooth. The Banach space $E$ is said to be strictly convex if $\left\|\frac{x+y}{2}\right\|<1$ whenever $x, y \in S_{E}$ and $x \neq y$. It is well known that $E$ is uniformly convex if and only if $E^{*}$ is uniformly smooth. It is also known that if $E$ is reflexive, then $E$ is strictly convex if and only if $E^{*}$ is smooth; for more details, see [13, 14].

Let $E$ be a smooth, strictly convex and reflexive Banach space, and let $J$ be the normalized duality mapping of $E$. Let $C$ be a nonempty closed convex subset of $E$. The generalized projection $\Pi_{C}$ from $E$ onto $C$ is denoted by

$$
\Pi_{C}(x)=\underset{y \in C}{\operatorname{argmin}} \phi(y, x)
$$

where $\phi(x, y)=\|x\|^{2}-2\langle x, J y\rangle+\|y\|^{2}$. If $E=H$ is a Hilbert space, then $\phi(x, y)=\|x-y\|^{2}$ for all $x, y \in H$.

Let $E$ be a Banach space with the norm $\|\cdot\|$ and the dual space $E^{*}$. A function $g: E \rightarrow$ $(-\infty,+\infty]$ is said to be proper if the domain $\operatorname{dom} g=\{x \in E: g(x)<\infty\}$ is nonempty. It is also called lower semicontinuous if $\{x \in E: g(x) \leq r\}$ is closed for all $r \in \mathbb{R}$. We say that $g$ is upper semicontinuous if $\{x \in E: g(x) \geq r\}$ is closed for all $r \in \mathbb{R}$. The function $g$ is said to be convex if

$$
g(\alpha x+(1-\alpha) y) \leq \alpha g(x)+(1-\alpha) g(y)
$$

for all $x, y \in E$ and $\alpha \in(0,1)$. It is also said to be strictly convex if the strict inequality holds in (1.2) for all $x, y \in \operatorname{dom} g$ with $x \neq y$ and $\alpha \in(0,1)$.

For any convex function $g: E \rightarrow(-\infty,+\infty]$, we denote the domain of $g$ by dom $g=\{x \in$ $E: g(x)<\infty\}$. For any $x \in$ int dom $g$ and any $y \in E$, we denote by $g^{o}(x, y)$ the right-hand 
derivative of $g$ at $x$ in the direction $y$, that is,

$$
g^{o}(x, y)=\lim _{t \downarrow 0} \frac{g(x+t y)-g(x)}{t} .
$$

The function $g$ is said to be Gâteaux differentiable at $x$ if $\lim _{t \rightarrow 0} \frac{g(x+t y)-g(x)}{t}$ exists for any $y$. In this case, $g^{o}(x, y)$ coincides with $\nabla g(x)$, the value of the gradient $\nabla g$ of $g$ at $x$ (see, for example, [9, p.12] or [13, p.508]). A convex function $g: E \rightarrow \mathbb{R}$ is said to be Gâteaux differentiable if it is Gâteaux differentiable everywhere. Let $g: E \rightarrow \mathbb{R}$ be a convex and Gâteaux differentiable function. Then the Bregman distance $[15,16]$ corresponding to $g$ is the function $D_{g}: E \times E \rightarrow \mathbb{R}$ defined by

$$
D_{g}(x, y)=g(x)-g(y)-\langle x-y, \nabla g(y)\rangle, \quad \forall x, y \in E .
$$

It is clear that $D_{g}(x, y) \geq 0$ for all $x, y \in E$. In the case when $E$ is a smooth Banach space, setting $g(x)=\|x\|^{2}$ for all $x \in E$, we have $\nabla g(x)=2 J x$ for all $x \in E$, and hence

$$
\begin{aligned}
D_{g}(x, y) & =\|x\|^{2}-\|y\|^{2}-\langle x-y, \nabla g(y)\rangle \\
& =\|x\|^{2}-\|y\|^{2}-\langle x-y, 2 J y\rangle \\
& =\|x\|^{2}-\|y\|^{2}-\langle x, 2 J y\rangle+2\|y\|^{2} \\
& =\|x\|^{2}-2\langle x, J y\rangle+\|y\|^{2} \\
& =\phi(x, y)
\end{aligned}
$$

for all $x, y \in E$.

The theory of fixed points with respect to Bregman distances have been studied in the last ten years and much intensively in the last four years. In [17], Bauschke and Combettes introduced an iterative method to construct the Bregman projection of a point onto a countable intersection of closed and convex sets in reflexive Banach spaces. They proved strong convergence theorem of the sequence produced by their method; for more detail, see [17, Theorem 4.7]. For some recent articles on the existence of fixed points for Bregman nonexpansive type mappings, we refer the readers to [17-26].

Let $E$ be a Banach space, and let $g: E \rightarrow \mathbb{R}$ be a convex and Gâteaux differentiable function. Let $C$ be a nonempty and closed convex subset of $E$. A mapping $T: C \rightarrow E$ is called nonexpansive if

$$
\|T x-T y\| \leq\|x-y\|, \quad \forall x, y \in C .
$$

The mapping $T: C \rightarrow E$ is called Bregman nonexpansive if

$$
D_{g}(T x, T y) \leq D_{g}(x, y), \quad \forall x, y \in C .
$$

Let us give an example of a Bregman nonexpansive mapping which is not a nonexpansive mapping (see also [27]). 
Example 1.1 Let $g: \mathbb{R} \rightarrow \mathbb{R}$ be a function defined by

$$
g(x)=x^{20}, \quad \forall x \in \mathbb{R} .
$$

We define a mapping $T:[0,0.9] \rightarrow[0,0.9]$ by

$$
T(x)=x^{2}, \quad \forall x \in[0,0.9]
$$

Then $T$ is not a nonexpansive mapping in the sense of (1.5), but it is a Bregman nonexpansive mapping relative to $D_{g}$ in the sense of (1.6). Indeed, taking $x=\frac{3}{4}$ and $y=\frac{1}{2}$, we see that $T$ is not a nonexpansive mapping in the sense of (1.5). Now, we show that

$$
D_{g}(T x, T y) \leq D_{g}(x, y), \quad \forall x, y \in[0,0.9] .
$$

Let $x \in[0,0.9]$ be fixed. We define a mapping $f:[0,0.9] \rightarrow[0,0.9]$ by

$$
f(y)=D_{g}(T x, T y)-D_{g}(x, y), \quad \forall y \in[0,0.9]
$$

Then

$$
\begin{aligned}
f(y) & =g(T x)-g(T y)-\langle T x-T y, \nabla g(T y)\rangle-[g(x)-g(y)-\langle x-y, \nabla(y)\rangle] \\
& =g(T x)-g(T y)-\left\langle T x-T y, g^{\prime}(T y)\right\rangle-\left[g(x)-g(y)-\left\langle x-y, g^{\prime}(y)\right\rangle\right] \\
& =x^{40}-20 x^{2} y^{38}+19 y^{40}-x^{20}-19 y^{20}+20 x y^{19} .
\end{aligned}
$$

This implies that

$$
\begin{aligned}
f^{\prime}(y) & =-760 x^{2} y^{37}+760 y^{39}-380 y^{19}+38 x y^{18} \\
& =380 y^{18}\left[-2 x^{2} y^{19}+2 y^{21}-y+x\right] \\
& =380 y^{18}\left[2 y^{19}\left(y^{2}-x^{2}\right)-(y-x)\right] \\
& =380 y^{18}(y-x)\left[2 y^{19}(y+x)-1\right] .
\end{aligned}
$$

Since $x$ and $y$ are in $[0,0.9]$, we obtain

$$
2 y^{19}(y+x)-1<2(0.9)^{19}(0.9+0.9)-1<0 .
$$

Therefore, $f^{\prime}(y) \geq 0$ if $y \leq x$ and $f^{\prime}(y) \leq 0$ if $y>x$. Moreover, $f(y)=0$ if $x=y$. Hence, $f(y) \leq 0$ for all $y \in[0,0.9]$, which implies that

$$
D_{g}(T x, T y) \leq D_{g}(x, y), \quad \forall x, y \in[0,0.9] .
$$

In this paper we establish some common fixed point results for the Banach operator and symmetric Banach operator pairs in reflexive Banach spaces for Bregman nonexpansive mappings that generalize the concept of nonexpansivity. Our results improve and generalize many known results in the current literature; see, for example, [2]. 


\section{Basic definitions and results}

Let $E$ be a real Banach space. Let $g: E \rightarrow \mathbb{R}$ be a convex and Gâteaux differentiable function. For any $x \in E$ and $r>0$, we define the Bregman ball centered at $x$ with radius $r$ by

$$
B(x, r)=\left\{y \in E: D_{g}(x, y)<r\right\} .
$$

The Bregman closed ball centered at $x$ with radius $r$ is denoted by

$$
\bar{B}(x, r)=\left\{y \in E: D_{g}(x, y) \leq r\right\} .
$$

Recall that a subset $C$ of a real Banach space $E$ is Bregman admissible if it is a nonempty intersection of Bregman closed balls. The class of all Bregman admissible subsets of $C$ is denoted by $\mathcal{B} \mathcal{A}(C)$.

Remark 2.1 Let $E$ be a real Banach space. Let $g: E \rightarrow \mathbb{R}$ be a continuous, convex and Gâteaux differentiable function. Then, for any $x \in E$ and $r>0$, any Bregman closed ball centered at $x$ with radius $r$ is $\tau\left(D_{g}\right)$ closed, where $\tau\left(D_{g}\right)$ is the topology induced by $D_{g}$ on $E$. Indeed, suppose $\left\{y_{n}\right\}_{n \in \mathbb{N}} \subset \bar{B}(x, r)$ is a sequence such that $y_{n} \rightarrow y \in E$ as $n \rightarrow \infty$. Since $g$ is continuous, so we have $g\left(y_{n}\right) \rightarrow g(y)$. This, together with the definition of the Bregman distance (see (1.4)), implies that

$$
\lim _{n \rightarrow \infty}\left|D_{g}\left(x, y_{n}\right)-D_{g}(x, y)\right|=0
$$

Thus we have $D_{g}(x, y) \leq r$. We refer the readers to see some details on quasipseudometric concept in [28].

At this point we introduce some notation which will be used throughout the remainder of this work. For a subset $A$ of $E$, we set

$$
\begin{aligned}
& B r_{x}(A)=\sup \left\{D_{g}(x, y): y \in A\right\}, \quad x \in E ; \\
& B R(A)=\inf \left\{B r_{x}(A): x \in A\right\} ; \\
& \text { B-diam }(A)=\sup \left\{D_{g}(x, y): x, y \in A\right\} ; \\
& B C_{A}(A)=\left\{x \in A: B r_{x}(A)=B R(A)\right\} ; \\
& \operatorname{cov}(A)=\cap\{B: B \text { is a Bregman ball and } B \supseteq A\} .
\end{aligned}
$$

B-diam $(A)$ is called the Bregman diameter of $A, B R(A)$ is called the Bregman Chebyshev radius of $A, B C_{A}(A)$ is called the Bregman Chebyshev center of $A$ and $\operatorname{cov}(A)$ is called the cover of $A$.

Definition 2.1 Let $\mathcal{F}$ be a convexity structure on $E$.

(i) We will say that $\mathcal{F}$ is compact if any family $\left(A_{\alpha}\right)_{\alpha \in \Gamma}$ of elements of $\mathcal{F}$ has a nonempty intersection provided $\bigcap_{\alpha \in F} A_{\alpha} \neq \emptyset$ for any finite subset $F \subset \Gamma$;

(ii) We will say that $\mathcal{F}$ is normal if for any $A \in \mathcal{F}$, not reduced to one point, we have $B R(A)<\mathrm{B}-\operatorname{diam}(A)$. 
Definition 2.2 The ordered pair $(S, T)$ of two self-maps of a closed and convex subset $C$ of a Banach space $E$ is called a Banach operator pair if the set Fix $(T)$ is $S$-invariant, namely $S(\operatorname{Fix}(T)) \subseteq \operatorname{Fix}(T)$. The ordered pair $(S, T)$ is called nontrivially a Banach operator pair if $\operatorname{Fix}(T)$ is not empty and $(S, T)$ is a Banach operator pair.

Obviously, a commuting pair $(S, T)$ is a Banach operator pair but not conversely in general; see [4-16, 29-34].

Let $A: E \rightarrow 2^{E^{*}}$ be a set-valued mapping. We define the domain and range of $A$ by $\operatorname{dom} A=\{x \in E: A x \neq \emptyset\}$ and $\operatorname{ran} A=\bigcup_{x \in E} A x$, respectively. The graph of $A$ is denoted by $G(A)=\left\{\left(x, x^{*}\right) \in E \times E^{*}: x^{*} \in A x\right\}$. The mapping $A \subset E \times E^{*}$ is said to be monotone [35] if $\left\langle x-y, x^{*}-y^{*}\right\rangle \geq 0$ whenever $\left(x, x^{*}\right),\left(y, y^{*}\right) \in A$. It is also said to be maximal monotone [36] if its graph is not contained in the graph of any other monotone operator on $E$. If $A \subset E \times E^{*}$ is maximal monotone, then we can show that the set $A^{-1} 0=\{z \in E: 0 \in A z\}$ is closed and convex. For a proper, lower semicontinuous and convex function $g: E \rightarrow(-\infty,+\infty]$, the subdifferential $\partial g$ of $g$ is defined by

$$
\partial g(x)=\left\{x^{*} \in E^{*}: g(x)+\left\langle y-x, x^{*}\right\rangle \leq g(y), \forall y \in E\right\}
$$

for all $x \in E$. It is well known that $\partial g \subset E \times E^{*}$ is maximal monotone [37, 38]. For any proper, lower semicontinuous and convex function $g: E \rightarrow(-\infty,+\infty]$, the conjugate function $g^{*}$ of $g$ is defined by

$$
g^{*}\left(x^{*}\right)=\sup _{x \in E}\left\{\left\langle x, x^{*}\right\rangle-g(x)\right\}
$$

for all $x^{*} \in E^{*}$. It is well known that $g(x)+g^{*}\left(x^{*}\right) \geq\left\langle x, x^{*}\right\rangle$ for all $\left(x, x^{*}\right) \in E \times E^{*}$. It is also known that $\left(x, x^{*}\right) \in \partial g$ is equivalent to

$$
g(x)+g^{*}\left(x^{*}\right)=\left\langle x, x^{*}\right\rangle .
$$

We also know that if $g: E \rightarrow(-\infty,+\infty]$ is a proper, lower semicontinuous and convex function, then $g^{*}: E^{*} \rightarrow(-\infty,+\infty]$ is a proper, weak ${ }^{*}$ lower semicontinuous and convex function; see [14] for more details on convex analysis. Let $g: E \rightarrow \mathbb{R}$ be a convex function. The function $g$ is also said to be Fréchet differentiable at $x \in E$ (see, for example, [29, p.13] or [30, p.508]) if for all $\epsilon>0$, there exists $\delta>0$ such that $\|y-x\| \leq \delta$ implies that

$$
|g(y)-g(x)-\langle y-x, \nabla g(x)\rangle| \leq \epsilon\|y-x\| .
$$

A convex function $g: E \rightarrow \mathbb{R}$ is said to be Fréchet differentiable if it is Fréchet differentiable everywhere. It is well known that if a continuous convex function $g: E \rightarrow \mathbb{R}$ is Gâteaux differentiable, then $\nabla g$ is norm-to-weak* continuous (see, for example, [29, Proposition 1.1.10]). Also, it is known that if $g$ is Fréchet differentiable, then $\nabla g$ is norm-tonorm continuous (see, [30, p.508]). The mapping $\nabla g$ is said to be weakly sequentially continuous if $x_{n} \rightarrow x$ implies that $\nabla g\left(x_{n}\right) \rightarrow^{*} \nabla g(x)$ (for more details, see [29, Theorem 3.2.4] or [30, p.508]). The function $g$ is said to be strongly coercive if

$$
\lim _{\left\|x_{n}\right\| \rightarrow \infty} \frac{g\left(x_{n}\right)}{\left\|x_{n}\right\|}=\infty .
$$


It is also said to be bounded on bounded subsets if $g(U)$ is bounded for each bounded subset $U$ of $E$.

Remark 2.2 Let $E$ be a real Banach space. Let $g: E \rightarrow \mathbb{R}$ be a Gâteaux differentiable function which is bounded on bounded subsets. Let $A$ be a bounded subset of $E$. Then B- $\operatorname{diam}(A)=\sup \left\{D_{g}(x, y): x, y \in A\right\}<\infty$. Indeed, the function $g$ is bounded on bounded subsets of $E$ and, thus, $\nabla g$ is also bounded on bounded subsets of $E^{*}$ (see, for example, [29, Proposition 1.1.11] for more details). This implies that there exist positive real numbers $M_{1}, M_{2}$ and $M_{3}$ such that

$$
\sup \{|g(x)|: x \in A\} \leq M_{1}, \quad \sup \{\|x\|: x \in A\} \leq M_{2}
$$

and

$$
\sup \{\|\nabla g(z)\|: z \in A\} \leq M_{3} .
$$

It follows that for any $x, y \in A$,

$$
\begin{aligned}
D_{g}(x, y) & =g(x)-g(y)-\langle x-y, \nabla g(y)\rangle \\
& \leq|g(x)|+|g(y)|+\|x-y\|\|\nabla g(y)\| \\
& \leq 2 M_{1}+2 M_{2} M_{3} .
\end{aligned}
$$

Therefore, B-diam $(A)=\sup \left\{D_{g}(x, y): x, y \in A\right\}<\infty$.

The following definition is slightly different from that in Butnariu and Iusem [29].

Definition 2.3 [30] Let $E$ be a Banach space. The function $g: E \rightarrow \mathbb{R}$ is said to be a Bregman function if the following conditions are satisfied:

(1) $g$ is continuous, strictly convex and Gâteaux differentiable;

(2) the set $\left\{y \in E: D_{g}(x, y) \leq r\right\}$ is bounded for all $x \in E$ and $r>0$.

The following lemma follows from Butnariu and Iusem [29] and Zălinscu [39].

Lemma 2.1 Let $E$ be a reflexive Banach space and let $g: E \rightarrow \mathbb{R}$ be a strongly coercive Bregman function. Then

(1) $\nabla g: E \rightarrow E^{*}$ is one-to-one, onto and norm-to-weak ${ }^{*}$ continuous;

(2) $\langle x-y, \nabla g(x)-\nabla g(y)\rangle=0$ if and only if $x=y$;

(3) $\left\{x \in E: D_{g}(x, y) \leq r\right\}$ is bounded for all $y \in E$ and $r>0$;

(4) dom $g^{*}=E^{*}, g^{*}$ is Gâteaux differentiable and $\nabla g^{*}=(\nabla g)^{-1}$.

Let $E$ be a Banach space and let $C$ be a nonempty and convex subset of $E$. Let $g: E \rightarrow \mathbb{R}$ be a convex and Gâteaux differentiable function. Then we know from [40] that for $x \in E$ and $x_{0} \in C, D_{g}\left(x_{0}, x\right)=\min _{y \in C} D_{g}(y, x)$ if and only if

$$
\left\langle y-x_{0}, \nabla g(x)-\nabla g\left(x_{0}\right)\right\rangle \leq 0, \quad \forall y \in C .
$$


Further, if $C$ is a nonempty, closed and convex subset of a reflexive Banach space $E$ and $g: E \rightarrow \mathbb{R}$ is a strongly coercive Bregman function, then for each $x \in E$, there exists a unique $x_{0} \in C$ such that

$$
D_{g}\left(x_{0}, x\right)=\min _{y \in C} D_{g}(y, x)
$$

The Bregman projection proj $_{C}^{g}$ from $E$ onto $C$ is defined by $\operatorname{proj}_{C}^{g}(x)=x_{0}$ for all $x \in E$. It is also well known that proj ${ }_{C}^{g}$ has the following property:

$$
D_{g}\left(y, \operatorname{proj}_{C}^{g} x\right)+D_{g}\left(\operatorname{proj}_{C}^{g} x, x\right) \leq D_{g}(y, x)
$$

for all $y \in C$ and $x \in E$ (see [29] for more details).

Let $E$ be a Banach space and $B_{r}:=\{z \in E:\|z\| \leq r\}$ for all $r>0$. Then a function $g: E \rightarrow \mathbb{R}$ is said to be uniformly convex on bounded subsets ([39, pp.203-221]) if $\rho_{r}(t)>0$ for all $r, t>0$, where $\rho_{r}:[0,+\infty) \rightarrow[0, \infty]$ is defined by

$$
\rho_{r}(t)=\inf _{x, y \in B_{r},\|x-y\| t=t, \alpha \in(0,1)} \frac{\alpha g(x)+(1-\alpha) g(y)-g(\alpha x+(1-\alpha) y)}{\alpha(1-\alpha)}
$$

for all $t \geq 0$. The function $\rho_{r}$ is called the gage of uniform convexity of $g$. The function $g$ is also said to be uniformly smooth on bounded subsets ([39, pp.207-221]) if $\lim _{t \downarrow} \frac{\sigma_{r}(t)}{t}=0$ for all $r>0$, where $\sigma_{r}:[0,+\infty) \rightarrow[0, \infty]$ is defined by

$$
\sigma_{r}(t)=\sup _{x \in B_{r}, y \in S_{E}, \alpha \in(0,1)} \frac{\alpha g(x+(1-\alpha) t y)+(1-\alpha) g(x-\alpha t y)-g(x)}{\alpha(1-\alpha)}
$$

for all $t \geq 0$.

The function $g$ is said to be uniformly convex if the function $\delta_{g}:[0,+\infty) \rightarrow[0,+\infty]$, defined by

$$
\delta_{g}(t):=\sup \left\{\frac{1}{2} g(x)+\frac{1}{2} g(y)-g\left(\frac{x+y}{2}\right):\|y-x\|=t\right\},
$$

satisfies that $\lim _{t \downarrow 0} \frac{\sigma_{r}(t)}{t}=0$. Let $g: E \rightarrow(-\infty,+\infty]$ be a convex and Gâteaux differentiable function. Recall that, in view of [29, Section 1.2, p.17], the function $g$ is called totally convex at a point $x \in$ int dom $g$ if its modulus of total convexity at $x$, that is, the function $v_{g}:$ int $\operatorname{dom} g \times[0,+\infty) \rightarrow[0,+\infty)$ defined by

$$
v_{g}(x, t):=\inf \left\{D_{g}(y, x): y \in \operatorname{int} \operatorname{dom} g,\|y-x\|=t\right\},
$$

is positive whenever $t>0$. The function $g$ is called totally convex when it is totally convex at every point $x \in$ int dom $g$. Moreover, the function $g$ is called totally convex on bounded subsets if $v_{g}(x, t)>0$ for any bounded subset $X$ of $E$ and for any $t>0$, where the modulus of total convexity of the function $g$ on the set $X$ is the function $v_{g}$ : int $\operatorname{dom} g \times[0,+\infty) \rightarrow$ $[0,+\infty)$ defined by

$$
v_{g}(X, t):=\inf \left\{v_{g}(x, t): x \in X \cap \operatorname{int} \operatorname{dom} g\right\} .
$$


It is well known that any uniformly convex function is totally convex, but the converse is not true in general (see [29, Section 1.3, p.30]).

It is also well known that $g$ is totally convex on bounded sets if and only if the function $g$ is uniformly convex on bounded sets (see [41, Theorem 2.10, p.9]).

Examples of totally convex functions can be found, for instance, in [29, 41].

Let $E$ be a Banach space and let $g: E \rightarrow \mathbb{R}$ be a convex and Gâteaux differentiable function. Then the Bregman distance $[15,16]$ does not satisfy the well-known properties of a metric, but it does have the following important property, which is called the three point identity [42]:

$$
D_{g}(x, z)=D_{g}(x, y)+D_{g}(y, z)+\langle x-y, \nabla g(y)-\nabla g(z)\rangle, \quad \forall x, y, z \in E .
$$

In particular, it can be easily seen that

$$
D_{g}(x, y)=-D_{g}(y, x)+\langle x-y, \nabla g(x)-\nabla g(y)\rangle, \quad \forall x, y \in E .
$$

Indeed, by letting $z=x$ in (2.5) and taking into account that $D_{g}(x, x)=0$, we get the desired result.

We will need the following important result; for the proof, we refer to ([29, p.67]).

Lemma 2.2 Let $E$ be a Banach space and let $g: E \rightarrow \mathbb{R}$ be a Gâteaux differentiable function which is uniformly convex on bounded sets. Let $\left\{x_{n}\right\}_{n \in \mathbb{N}}$ and $\left\{y_{n}\right\}_{n \in \mathbb{N}}$ be bounded sequences in $E$. Then the following assertions are equivalent:

(1) $\lim _{n \rightarrow \infty} D_{g}\left(x_{n}, y_{n}\right)=0$;

(2) $\lim _{n \rightarrow \infty}\left\|x_{n}-y_{n}\right\|=0$.

Remark 2.3 Let $E$ be a Banach space and let $g: E \rightarrow \mathbb{R}$ be a convex and Gâteaux differentiable function. Let $C$ be a closed and convex subset of $E$. Then, in view of Lemma 2.2, any Bregman nonexpansive mapping $T: C \rightarrow C$ is continuous.

Let $l^{\infty}$ denote the Banach space of bounded real sequences with the supremum norm. It is well known that there exists a bounded linear functional $\mu$ on $l^{\infty}$ such that the following three conditions hold:

(1) If $\left\{t_{n}\right\}_{n \in \mathbb{N}} \in l^{\infty}$ and $t_{n} \geq 0$ for every $n \in \mathbb{N}$, then $\mu\left(t_{n}\right) \geq 0$;

(2) If $t_{n}=1$ for every $n \in \mathbb{N}$, then $\mu\left(t_{n}\right)=1$;

(3) $\mu\left(\left\{t_{n+1}\right\}\right)=\mu\left(\left\{t_{n}\right\}\right)$ for all $\left\{t_{n}\right\}_{n \in \mathbb{N}} \in l^{\infty}$.

Such a functional $\mu$ is called a Banach limit and the value of $\mu$ at $\left\{t_{n}\right\}_{n \in \mathbb{N}} \in l^{\infty}$ is denoted by $\mu_{n} t_{n}$ (see, for example, [13]).

\section{Common fixed points for Banach operator pairs}

Let $E$ be a Banach space and let $g: E \rightarrow \mathbb{R}$ be a convex and Gâteaux differentiable function. Let $C$ be a closed and convex subset of a real Banach space $E$. A mapping $T: C \rightarrow E$ is said to be Bregman quasi-nonexpansive [17] if $F(T) \neq \emptyset$ and

$$
D_{g}(p, T x) \leq D_{g}(p, x), \quad \forall x \in C, p \in F(T) .
$$


Let $C$ and $D$ be nonempty subsets of a real Banach space $E$ with $D \subset C$. A mapping $R_{D}$ : $C \rightarrow D$ is said to be sunny if

$$
R_{D}\left(R_{D} x+t\left(x-R_{D} x\right)\right)=R_{D} x
$$

for each $x \in E$ and $t \geq 0$. A mapping $R_{D}: C \rightarrow D$ is said to be a retraction if $R_{D} x=x$ for each $x \in C$.

The following result was proved in [24].

Lemma 3.1 Let $E$ be a reflexive Banach space and let $g: E \rightarrow \mathbb{R}$ be a convex, continuous, strongly coercive and Gâteaux differentiable function which is bounded on bounded subsets and uniformly convex on bounded subsets. Let $C$ be a nonempty, closed and convex subset of E. Let $T: C \rightarrow E$ be a Bregman quasi-nonexpansive mapping. Then $F(T)$ is closed and convex.

Corollary 3.1 Let E be a reflexive Banach space and let $g: E \rightarrow \mathbb{R}$ be a convex, continuous, strongly coercive and Gâteaux differentiable function which is bounded on bounded sets and uniformly convex on bounded sets. Let $C$ be a nonempty, closed and convex subset of $E$ and let $T: C \rightarrow E$ be a Bregman nonexpansive mapping. If $F(T) \neq \emptyset$, then it is closed and convex.

Using ideas in [43], we can prove the following result.

Theorem 3.1 Let $E$ be a reflexive Banach space and let $g: E \rightarrow \mathbb{R}$ be a convex, continuous, strongly coercive and Gâteaux differentiable function which is bounded on bounded subsets and uniformly convex on bounded subsets. Let $C$ be a nonempty, closed and convex subset of $E$ and let $T: C \rightarrow C$ be a mapping. Let $\left\{x_{n}\right\}_{n \in \mathbb{N}}$ be a bounded sequence of $C$ and let $\mu$ be a mean on $l^{\infty}$. Suppose that

$$
\mu_{n} D_{g}\left(x_{n}, T y\right) \leq \mu_{n} D_{g}\left(x_{n}, y\right)
$$

for all $y \in C$. Then $T$ has a fixed point in $C$.

Proof Let $\mu$ be a mean on $l^{\infty}$ and $\left\{x_{n}\right\}_{n \in \mathbb{N}}$ be a bounded sequence in $C$. Define a mapping $h: E^{*} \rightarrow \mathbb{R}$ by

$$
h\left(x^{*}\right)=\mu_{n}\left\langle x_{n}, x^{*}\right\rangle, \quad x^{*} \in E^{*} .
$$

Since $\mu$ is linear, so is $h$. Observe that

$$
\begin{aligned}
\left|h\left(x^{*}\right)\right| & \left.=\left|\mu_{n}\right| x_{n}, x^{*}\right\rangle \mid \\
& \leq\|\mu\| \sup _{n \in \mathbb{N}}\left|\left\langle x_{n}, x^{*}\right\rangle\right| \\
& \leq\|\mu\| \sup _{n \in \mathbb{N}}\left\|x_{n}\right\|\left\|x^{*}\right\| \\
& =\sup _{n \in \mathbb{N}}\left\|x_{n}\right\|\left\|x^{*}\right\|
\end{aligned}
$$


for all $x^{*} \in E^{*}$. This implies that $h$ is a linear and continuous real-valued mapping on $E^{*}$. Since $E$ is reflexive, then there exists a unique element $z \in E$ such that

$$
h\left(x^{*}\right)=\mu_{n}\left\langle x_{n}, x^{*}\right\rangle=\left\langle z, x^{*}\right\rangle, \quad x^{*} \in E^{*} .
$$

We claim that $z \in C$. If not, then by the separation theorem [13] there exists $y^{*} \in E^{*}$ such that

$$
\left\langle z, y^{*}\right\rangle<\inf _{y \in C}\left\langle y, y^{*}\right\rangle
$$

Since $\left\{x_{n}\right\}_{n \in \mathbb{N}} \subset C$, we conclude that

$$
\left\langle z, y^{*}\right\rangle<\inf _{y \in C}\left\langle y, y^{*}\right\rangle \leq \inf _{n \in \mathbb{N}}\left\langle x_{n}, y^{*}\right\rangle \leq \mu_{n}\left\langle x_{n}, x^{*}\right\rangle=\left\langle z, x^{*}\right\rangle .
$$

This is a contradiction. Thus we have $z \in C$. In view of (2.5), for any $y \in C$ and $n \in \mathbb{N}$, we deduce that

$$
D_{g}\left(x_{n}, y\right)=D_{g}\left(x_{n}, T y\right)+D_{g}(T y, y)+\left\langle x_{n}-T y, \nabla g(T y)-\nabla g(y)\right\rangle .
$$

Thus we have, for any $y \in C$, that

$$
\begin{aligned}
\mu_{n} D_{g}\left(x_{n}, y\right) & =\mu_{n} D_{g}\left(x_{n}, T y\right)+\mu_{n} D_{g}(T y, y)+\mu_{n}\left\langle x_{n}-T y, \nabla g(T y)-\nabla g(y)\right\rangle \\
& =\mu_{n} D_{g}\left(x_{n}, T y\right)+D_{g}(T y, y)+\langle z-T y, \nabla g(T y)-\nabla g(y)\rangle .
\end{aligned}
$$

By the assumption, we have that

$$
\mu_{n} D_{g}\left(x_{n}, T y\right) \leq \mu_{n} D_{g}\left(x_{n}, y\right)
$$

for all $y \in C$. This implies that

$$
\mu_{n} D_{g}\left(x_{n}, y\right) \leq \mu_{n} D_{g}\left(x_{n}, y\right)+D_{g}(T y, y)+\langle z-T y, \nabla g(T y)-\nabla g(y)\rangle
$$

for all $y \in C$. Putting $y=z$ in (3.1) and taking into account (2.6), we see that

$$
\begin{aligned}
0 & \leq D_{g}(T z, z)+\langle z-T z, \nabla g(T z)-\nabla g(z)\rangle \\
& =-D_{g}(z, T z)+\langle z-T z, \nabla g(z)-\nabla g(T z)\rangle+\langle z-T y, \nabla g(T z)-\nabla g(z)\rangle \\
& =-D_{g}(z, T z) .
\end{aligned}
$$

Then we have $0 \leq-D_{g}(z, T z)$, which implies that $D_{g}(z, T z)=0$. In view of Lemma 2.2, we conclude that $T z=z$, which completes the proof.

Remark 3.1 Let $g$ and $T$ be as in Example 1.1. Let $x \in[0,0.9]$ be fixed. Then $\left\{T^{n} x\right\}_{n \in \mathbb{N}}$ is a bounded sequence in $[0,0.9]$. Set $x_{n}:=T^{n} x$ for $n=1,2, \ldots$ It is obvious that $T$ satisfies all the aspects of the hypothesis of Theorem 3.1, so it has a fixed point. 
Corollary 3.2 Let $E$ be a reflexive Banach space and let $g: E \rightarrow \mathbb{R}$ be a convex, continuous, strongly coercive and Gâteaux differentiable function which is bounded on bounded subsets and uniformly convex on bounded subsets. Let $C$ be a nonempty, closed and convex subset of $E$ and let $T: C \rightarrow C$ be a mapping. Suppose that there exist $x \in C$ and a Banach limit $\mu$ such that $\left\{T^{n} x\right\}_{n \in \mathbb{N}}$ is bounded and

$$
\mu_{n} D_{g}\left(T^{n} x, T y\right) \leq \mu_{n} D_{g}\left(T^{n} x, y\right)
$$

for all $y \in C$. Then $T$ has a fixed point.

Corollary 3.3 Let E be a reflexive Banach space and let $g: E \rightarrow \mathbb{R}$ be a convex, continuous, strongly coercive and Gâteaux differentiable function which is bounded on bounded subsets and uniformly convex on bounded subsets. Let $C$ be a nonempty, closed and convex subset of $E$ and let $T: C \rightarrow C$ be a Bregman nonexpansive mapping. Suppose that there exists $x \in C$ such that $\left\{T^{n} x\right\}_{n \in \mathbb{N}}$ is bounded. Then $T$ has a fixed point.

Proof Let $\mu$ a Banach limit on $l^{\infty}$ and $x \in C$ be such that $\left\{T^{n} x\right\}_{n \in \mathbb{N}}$ is bounded. Then we have

$$
\mu_{n} D_{g}\left(T^{n} x, T y\right)=\mu_{n} D_{g}\left(T^{n+1} x, T y\right) \leq \mu_{n} D_{g}\left(T^{n} x, y\right)
$$

for all $y \in C$. In view of Corollary 3.2, we deduce that $F(T) \neq \emptyset$, which completes the proof.

Corollary 3.4 Let $E$ be a reflexive Banach space and let $g: E \rightarrow \mathbb{R}$ be a convex, continuous, strongly coercive and Gâteaux differentiable function which is bounded on bounded sets and uniformly convex on bounded sets. Let $C$ be a nonempty, bounded, closed and convex subset of $E$ and let $T: C \rightarrow C$ be a Bregman nonexpansive mapping. Then $T$ has a fixed point.

Definition 3.1 Let $A$ and $C$ be nonempty subsets of a real Banach space $E$ with $A \subset C$. We say that $A$ is a Bregman nonexpansive retract of $C$ if there exists a Bregman nonexpansive map $R: C \rightarrow A$ such that $R(a)=a$ for every $a \in A$.

Definition 3.2 Let $C$ be a nonempty, closed and convex subset of a real Banach space $E$. The mapping $T: C \rightarrow C$ is called Bregman NR-map if $\operatorname{Fix}(T)$ is a Bregman nonexpansive retract of $C$.

Theorem 3.2 Let $E$ be a reflexive Banach space and let $g: E \rightarrow \mathbb{R}$ be a convex, continuous, strongly coercive and Gâteaux differentiable function which is bounded on bounded sets and uniformly convex on bounded sets. Let $C$ be a nonempty, bounded, closed and convex subset of E. Let $T: C \rightarrow C$ be a continuous Bregman NR-map. Let $S: C \rightarrow C$ be a Bregman nonexpansive mapping such that $(S, T)$ is a Banach operator pair. Then $F(S, T)$ is not empty.

Proof Since the retract of a nonempty space is nonempty, $\operatorname{Fix}(T)$ is nonempty and is closed as $T$ is continuous. Since $T$ is a Bregman $N R$-map, then there exists a Bregman nonexpansive retract $R: C \rightarrow \operatorname{Fix}(T)$. Since $(S, T)$ is a Banach operator pair, then $S(\operatorname{Fix}(T)) \subset \operatorname{Fix}(T)$. 
Hence $S \circ R: C \rightarrow C$ is a Bregman nonexpansive map such that $S \circ R(C) \subset \operatorname{Fix}(T)$. Corollary 3.4 implies the existence of a fixed point of $S \circ R$. Clearly, such a fixed point is a fixed point of $S$ which belongs to $\operatorname{Fix}(T)$. Hence $\operatorname{Fix}(T) \cap \operatorname{Fix}(S)=F(S, T)$ is not empty.

Example 3.1 Let $E$ be a reflexive and smooth Banach space and let $C$ be a closed and convex subset of $E$ such that $0 \in C$. Let $T: C \rightarrow E$ be defined as

$$
T(x)=-x, \quad x \in C .
$$

Then $T$ is a Bregman quasi-nonexpansive mapping with $g(x)=\frac{1}{2}\|x\|^{2}, \nabla g(x)=J x$ for all $x \in C$ and $F(T)=\{0\}$. Indeed, it is clear that

$$
\|T x\| \leq\|x\|, \quad \forall x \in C
$$

This implies that

$$
g(T x)-g(x)=\frac{1}{2}\|T x\|^{2}-\frac{1}{2}\|x\|^{2} \leq\langle 0, \nabla g(T x)-\nabla g(x)\rangle=\langle p, \nabla g(T x)-\nabla g(x)\rangle
$$

for all $p \in F(T)$. Then we have

$$
\|p\|^{2}+\|T x\|^{2}-2\langle p, \nabla g(T x)\rangle \leq\|p\|^{2}+\|x\|^{2}-2\langle p, \nabla g(x)\rangle .
$$

This means that

$$
D_{g}(p, T x) \leq D_{g}(p, x)
$$

for all $p \in F(T)$ and $x \in C$. Hence, $T$ is a Bregman quasi-nonexpansive mapping. Define a mapping $R: C \rightarrow\{0\}$ by

$$
R(x)=0, \quad \forall x \in C .
$$

Then $T$ is a Bregman NR-map.

Assume now that $h: E \rightarrow \mathbb{R}$ is a lower semicontinuous function satisfying the following conditions:

(i) $h$ is totally convex on bounded sets;

(ii) $h$, as well as its Fenchel conjugate $h^{*}$, are defined and (Gâteaux) differentiable on $E$ and $E^{*}$, respectively;

(iii) $h^{\prime}$ is uniformly continuous and $h^{*}$ is bounded on bounded sets.

Let $A: \operatorname{dom} A \rightarrow E^{*}$ be an operator and $\Omega$ be a nonempty subset of $\operatorname{dom} A$ such that $0 \in \Omega$, $A(0)=0$ and $C \subset \operatorname{dom} A$. For any $\alpha \in(0, \infty)$, we define the operator $A_{\alpha}^{h}: \operatorname{dom} A \rightarrow E$ by

$$
A_{\alpha}^{h} x=h^{* \prime}\left(h^{\prime}(x)-\alpha A x\right) .
$$

It is worth mentioning that $A x=0$ if and only if $x \in \operatorname{dom} A$ is a fixed point of $A_{\alpha}^{h}$. The operator $A$ is said to be inverse-strongly-monotone relative to $h$ on the set $\Omega$ if there exist a real number $\alpha>0$ and a vector $z \in \Omega$ such that

$$
\left\langle A y, A_{\alpha}^{h} y-z\right\rangle \geq 0, \quad \forall y \in \Omega
$$


If we set $S:=A_{\alpha}^{h}$, then $S$ is a Bregman nonexpansive mapping (for more details, see [41]). It is clear that $T$ and $S$ satisfy all the aspects of the hypothesis of Theorem 3.2 and $T$ and $S$ have a common fixed point.

Remark 3.2 Let $E$ be a reflexive Banach space and let $g: E \rightarrow \mathbb{R}$ be a convex, continuous, strongly coercive and Gâteaux differentiable function which is bounded on bounded subsets and uniformly convex on bounded subsets. Let $C$ be a nonempty, bounded, closed and convex subset of $E$ and let $T: C \rightarrow C$ be a Bregman nonexpansive mapping. Then, in view of Corollary 3.4 and Lemma 3.1, $\operatorname{Fix}(T)$ is not empty and closed convex which implies that $\operatorname{Fix}(T)$ is a Bregman nonexpansive retract of $C$. Thus $T$ is a Bregman NR-map.

Theorem 3.3 Let $E$ be a reflexive Banach space and let $g: E \rightarrow \mathbb{R}$ be a convex, continuous, strongly coercive and Gâteaux differentiable function which is bounded on bounded subsets and uniformly convex on bounded subsets. Let $T$ and $S$ be two Bregman nonexpansive selfmappings defined on a closed and convex subset $C$ of $E$. If $(S, T)$ is a Banach operator pair and $T(C)$ is bounded, then $\operatorname{Fix}(T) \cap \operatorname{Fix}(S) \neq \emptyset$.

Proof Let $K=\overline{\operatorname{conv}}(T(C))$. Then $T: K \rightarrow K$ and $K$ is nonempty and bounded. In view of Corollary 3.4, the fixed point set $\operatorname{Fix}(T)$ of $T$ is nonempty and bounded. Since $(S, T)$ is a Banach operator pair, $S: \operatorname{Fix}(T) \rightarrow \operatorname{Fix}(T)$. By Corollary $3.4, S$ has a fixed point in $\operatorname{Fix}(T)$ as required.

The following slight extension of Theorem 3.3 can be proved easily.

Theorem 3.4 Let $E$ be a reflexive Banach space and let $g: E \rightarrow \mathbb{R}$ be a convex, continuous, strongly coercive and Gâteaux differentiable function which is bounded on bounded subsets and uniformly convex on bounded subsets. Let $C$ be a nonempty, closed and convex subset of $E$. Let $X$ be a normed space and $T$ and $S$ be two Bregman nonexpansive self-mappings defined on a closed convex set $C \subset E$. If $(S, T)$ is a Banach operator pair, and if $\overline{T^{n}(C)}$ is bounded for some $n \in \mathbb{N}$, then $\operatorname{Fix}(T) \cap \operatorname{Fix}(S) \neq \emptyset$.

Corollary 3.5 Let $E$ be a reflexive Banach space and let $g: E \rightarrow \mathbb{R}$ be a convex, continuous, strongly coercive and Gâteaux differentiable function which is bounded on bounded subsets and uniformly convex on bounded subsets. Let $C$ be a nonempty, bounded, closed and convex subset of E. Let $T: C \rightarrow C$ be Bregman nonexpansive. Let $S: C \rightarrow C$ be a Bregman nonexpansive mapping such that $(S, T)$ is a Banach operator pair. Then $F(S, T)$ is not empty.

Corollary 3.6 Let $E$ be a reflexive Banach space and let $g: E \rightarrow \mathbb{R}$ be a convex, continuous, strongly coercive and Gâteaux differentiable function which is bounded on bounded subsets and uniformly convex on bounded subsets. Let $C$ be a nonempty, closed and convex subset of E. Let $T: C \rightarrow C$ be a Bregman nonexpansive map such that $T(C)$ is bounded and $T(C) \subset$ Fix $(T)$. Let $S: C \rightarrow C$ be a Bregman nonexpansive mapping such that $(S, T)$ is nontrivially a Banach operator pair. Then $\operatorname{Fix}(S) \cap \operatorname{Fix}(T)$ is not empty.

Corollary 3.7 Let $E$ be a reflexive Banach space and let $g: E \rightarrow \mathbb{R}$ be a convex, continuous, strongly coercive and Gâteaux differentiable function which is bounded on bounded 
subsets and uniformly convex on bounded subsets. Let $C$ be a nonempty, closed and convex subset of E. Let $S, T: C \rightarrow C$ be a nontrivially Banach operator pair such that $\operatorname{Fix}(T)$ is bounded and $S$ is a Bregman nonexpansive map. Assume that $T: C \rightarrow \operatorname{Fix}(T)$ is a Bregman nonexpansive map. Then $\operatorname{Fix}(S) \cap \operatorname{Fix}(T)$ is not empty.

Theorem 3.5 Let $E$ be a reflexive Banach space and let $g: E \rightarrow \mathbb{R}$ be a convex, continuous, strongly coercive and Gâteaux differentiable function which is bounded on bounded subsets and uniformly convex on bounded subsets. Let $C$ be a nonempty, closed and convex subset of $E$ which has the property that every Bregman nonexpansive mapping of $C \rightarrow C$ is Bregman NR-map. Suppose $T: C \rightarrow C$ is a mapping for which $T^{n}$ is Bregman nonexpansive for some $n \in \mathbb{N}$, and suppose the restriction of $T$ to $\operatorname{Fix}\left(T^{n}\right)$ is also Bregman nonexpansive. Then $\operatorname{Fix}(T)$ is a nonempty Bregman nonexpansive retract of $C$. Consequently, if $S: C \rightarrow C$ is Bregman nonexpansive and if $(S, T)$ is a Banach operator pair, then $\operatorname{Fix}(T) \cap \operatorname{Fix}(S)$ is a nonempty Bregman nonexpansive retract of $C$.

Proof By assumption, there exists a Bregman nonexpansive retraction $R_{1}$ of $C$ onto $\operatorname{Fix}\left(T^{n}\right)$. Consequently, $T \circ R_{1}$ is a Bregman nonexpansive mapping of $C$ into $C$, so $\operatorname{Fix}\left(T \circ R_{1}\right)$ is a nonempty Bregman nonexpansive retract of $C$. But $x \in \operatorname{Fix}\left(T \circ R_{1}\right) \Leftrightarrow$ $x \in \operatorname{Fix}(T) \cap \operatorname{Fix}\left(T^{n}\right)$, and by Lemma $1[44]$

$$
x \in \operatorname{Fix}(T) \cap \operatorname{Fix}\left(T^{n}\right) \quad \Leftrightarrow \quad x \in \operatorname{Fix}\left(T^{n+1}\right) \cap \operatorname{Fix}\left(T^{n}\right)=\operatorname{Fix}(T) .
$$

Therefore there is a Bregman nonexpansive retraction $R_{2}$ of $C$ onto $\operatorname{Fix}(T)$. So, $S \circ R_{2}$ is a Bregman nonexpansive mapping of $C$ into $\operatorname{Fix}(T)$. Therefore $\operatorname{Fix}\left(S \circ R_{2}\right)=\operatorname{Fix}(S)=$ $\operatorname{Fix}(S) \cap \operatorname{Fix}(T)$ is a nonempty Bregman nonexpansive retract of $C$.

We might observe that in the above theorem it is not necessary that $T$ be Bregman nonexpansive. The only facts needed for the proof is that Fix $\left(T^{n}\right)$ be a Bregman nonexpansive retract of $C$.

\section{Fixed point of Banach operator family}

Definition 4.1 Let $C$ be a closed and convex subset of a real Banach space $E$ and let $T$ and $S$ be two self-maps on $C$. The pair $(S, T)$ is called a symmetric Banach operator pair if both $(S, T)$ and $(T, S)$ are Banach operator pairs, i.e., $T(\operatorname{Fix}(S)) \subseteq \operatorname{Fix}(S)$ and $S(\operatorname{Fix}(T)) \subseteq \operatorname{Fix}(T)$.

It is easy to see that the pair $(S, T)$ is a symmetric Banach operator pair if and only if $T$ and $S$ are commuting on $\operatorname{Fix}(T) \cup \operatorname{Fix}(S)$.

Definition 4.2 A subset $A$ of a Banach space $E$ is said to be a 1-local Bregman retract of $E$ if for every family $\left\{B_{i}: i \in I\right\}$ of Bregman closed balls centered in $A$ with nonempty intersection, it is the case that $A \cap\left(\bigcap_{i \in I} B_{i}\right) \neq \emptyset$. It is immediate that each Bregman nonexpansive retract of $E$ is a 1-local Bregman retract (but not conversely).

Definition 4.3 Let $C$ be a closed and convex subset of a real Banach space $E$ and let $\mathcal{T}$ be a family of mappings defined on $C$. Then the family $\mathcal{T}$ has a common fixed point if it is the fixed point of each member of $\mathcal{T}$. The family $\mathcal{T}$ is called a Banach operator family if any two of maps in the family form a symmetric Banach operator pair. 
Theorem 4.1 Let $E$ be a reflexive Banach space and let $g: E \rightarrow \mathbb{R}$ be a convex, continuous, strongly coercive and Gâteaux differentiable function which is bounded on bounded subsets and uniformly convex on bounded subsets. Let $C$ be a nonempty, closed and convex subset of $E$ and let $\mathcal{H}$ be a nonempty family of Bregman nonexpansive maps of $C$ into itself. If $\mathcal{H}$ is a Banach operator family and there exists $T \in \mathcal{H}$ such that $\overline{T(C)}$ is compact, then $\mathcal{H}$ has a common fixed point in $C$.

Proof Let $K=\overline{\operatorname{conv}}(T(C))$. It suffices to show that each finite subfamily of $\mathcal{H}$ has a nonempty common fixed point set in $K$. The full conclusion then follows from the compactness of $K$. Let $\left\{T_{1}, T_{2}, \ldots, T_{n}\right\}$ be a finite subfamily of $\mathcal{H}$. As above, $\operatorname{Fix}(T)$ is nonempty. Since $\left(T_{1}, T\right)$ is a Banach operator pair, $T_{1}: \operatorname{Fix}(T) \rightarrow \operatorname{Fix}(T)$. By Corollary 3.4, $T_{1}$ has a fixed point in $\operatorname{Fix}(T)$. Since $\left(T_{2}, T_{1}\right)$ is a Banach operator pair, $T_{2}: \operatorname{Fix}\left(T_{1}\right) \rightarrow \operatorname{Fix}\left(T_{1}\right)$. Proceeding in a step by step way, we conclude $\operatorname{Fix}(T) \cap \operatorname{Fix}\left(T_{1}\right) \cap \cdots \cap \operatorname{Fix}\left(T_{n}\right) \neq \emptyset$.

Theorem 4.2 Let $E$ be a reflexive Banach space and let $g: E \rightarrow \mathbb{R}$ be a convex, continuous, strongly coercive and Gâteaux differentiable function which is bounded on bounded subsets and uniformly convex on bounded subsets. Let $C$ be a nonempty, bounded, closed and convex subset of $E$ such that $\mathcal{B A}(C)$ is compact and normal. Let $\mathcal{T}$ be a family of Bregman nonexpansive mappings $T_{1}, T_{2}, \ldots, T_{n}, T_{i}: C \rightarrow C$. Assume that any two mappings from $\mathcal{T}$ form a symmetric Banach operator pair. Then the family $\mathcal{T}$ has a common fixed point. Moreover, the common fixed point set $\operatorname{Fix}(\mathcal{T})$ is a 1-local Bregman retract of $C$.

Proof First, let us prove that $F=\operatorname{Fix}(\mathcal{T})$ is not empty. Using Corollary 3.4, we know that $\operatorname{Fix}\left(T_{1}\right)$ is not empty. Since $\operatorname{Fix}\left(T_{1}\right)$ is a 1-local Bregman retract [4] of $C$, by a similar argument as in [4], we conclude that $\mathcal{A}\left(\operatorname{Fix}\left(T_{1}\right)\right)$ is compact and normal. On the other hand, we have $T_{2}\left(\operatorname{Fix}\left(T_{1}\right)\right) \subset \operatorname{Fix}\left(T_{1}\right)$ because any two mappings from $\mathcal{T}$ form a symmetric Banach operator pair. Hence $T_{2}$ has a fixed point in $\operatorname{Fix}\left(T_{1}\right)$. If we restrict ourselves to $\operatorname{Fix}\left(T_{1}, T_{2}\right)$, the common fixed point set of $T_{1}$ and $T_{2}$, then one can prove in an identical argument that $T_{3}$ has a fixed point in $\operatorname{Fix}\left(T_{1}, T_{2}\right)$. Step by step, we can prove that the common fixed point set $\operatorname{Fix}(\mathcal{T})$ of $T_{1}, \ldots, T_{n}$ is not empty. The same argument, used to prove that the fixed point set of a Bregman nonexpansive map is a 1-local Bregman retract, can be reproduced here to prove that $\operatorname{Fix}(\mathcal{T})$ is a 1-local Bregman retract.

Theorem 4.3 Let $E$ be a reflexive Banach space and let $g: E \rightarrow \mathbb{R}$ be a convex, continuous, strongly coercive and Gâteaux differentiable function which is bounded on bounded subsets and uniformly convex on bounded subsets. Let $C$ be a nonempty, bounded, closed and convex subset of $E$ such that $\mathcal{B A}(C)$ is compact and normal. Let $\mathcal{T}$ be a family of Bregman nonexpansive mappings $\left(T_{i}\right)_{i \in I}, T_{i}: C \rightarrow C$. Assume that any two mappings from $\mathcal{T}$ form a symmetric Banach operator pair. Then the family $\mathcal{T}$ has a common fixed point. Moreover, the common fixed point set $\operatorname{Fix}(\mathcal{T})$ is a 1-local Bregman retract of $C$.

Proof $\Gamma=2^{I}=\{\beta \subset I: \beta$ is finite and nonempty $\}$. It is obvious that $\Gamma$ is downward directed (the order on $\Gamma$ is the set inclusion). Theorem 4.2 implies that for every $\beta \in \Gamma$, the set $F_{\beta}$ of a common fixed point set of the mappings $T_{i}, i \in \beta$, is a nonempty 1-local Bregman retract of $C$. Clearly, the family $\left(F_{\beta}\right)_{\beta \in \Gamma}$ is decreasing. Using the remark following Theorem 6 [4], we deduce that $\bigcap_{\beta \in \Gamma} F_{\beta}$ is nonempty and is a 1-local Bregman retract of $C$. 
Lemma 4.1 Let $E$ be a reflexive Banach space and let $g: E \rightarrow \mathbb{R}$ be a convex, continuous, strongly coercive and Gâteaux differentiable function which is bounded on bounded subsets and uniformly convex on bounded subsets. Let $C$ be a nonempty, closed and convex subset of $E$ such that $\mathcal{B A}(C)$ is compact and normal. Let $\mathcal{T}$ be a family of Bregman nonexpansive mappings defined on $C$. Let $\tau$ be a topology on $C$ for which the closed balls are $\tau$-closed. Assume that there exists a bounded subset $A \subset C$ with $\delta=\mathrm{B}-\operatorname{diam}(A)$ such that $C=\bigcap_{a \in A} \bar{B}(a, \delta)$, is 1-local Bregman retract of $C, A \subset \overline{T(A)}^{\tau}$, for any $T \in \mathcal{T}$, where $\overline{T(A)}^{\tau}$ is the $\tau$-closure of $T(A)$. Assume that any two mappings from $\mathcal{T}$ form a symmetric Banach operator pair. Then the family $\mathcal{T}$ has a common fixed point.

Proof Denote by $\delta=$ B-diam $(A)$. Consider the subset

$$
C=\bigcap_{a \in A} \bar{B}(a, \delta)
$$

Clearly, we have $A \subset C$. Let $T \in \mathcal{T}$, then

$$
T(C) \subset \bigcap_{a \in A} \bar{B}(T(a), \delta)
$$

since $T$ is Bregman nonexpansive. This implies

$$
T(A) \subset \bigcap_{c \in T(C)} \bar{B}(c, \delta) .
$$

Since the Bregman closed balls are $\tau$-closed, we get

$$
\overline{T(A)}^{\tau} \subset \bigcap_{c \in T(C)} \bar{B}(c, \delta) .
$$

Our assumption implies

$$
A \subset \bigcap_{c \in T(C)} \bar{B}(c, \delta) .
$$

Hence

$$
T(C) \subset \bigcap_{a \in A} \bar{B}(a, \delta)=C
$$

Since $C$ is bounded and is 1-local Bregman retract of $C$, so $\mathcal{B A}(C)$ is compact and normal and the theorem above implies that $\mathcal{T}$ has a common fixed point.

Definition 4.4 Let $C$ be nonempty, closed and convex subset of a Banach space $E$. Let $\mathcal{T}$ be a family of mappings defined on $C$. The family $\mathcal{T}$ is called a semigroup if $S \circ T \in \mathcal{T}$ whenever $S, T \in \mathcal{T}$. We will call the semigroup $\mathcal{T}$ an invertible semigroup if and only if any element in $\mathcal{T}$ is invertible and $T^{-1} \in \mathcal{T}$ for any $T \in \mathcal{T}$. For any $x \in C$, define the orbit of $x$ by

$$
\mathcal{T}(x)=\{T(x) ; T \in \mathcal{T}\}
$$


Theorem 4.4 Let $C$ be a nonempty, closed and convex subset of a Banach space $E$ such that $\mathcal{B A}(C)$ is compact and normal. Let $\mathcal{T}$ be an invertible semigroup of isometric mappings defined on $H$ such that any two mappings from $\mathcal{T}$ form a symmetric Banach operator pair. Assume that $C=\bigcap_{a \in A} \bar{B}(a, \delta)$ is 1-local Bregman retract of $C$, where $A=\mathcal{T}(x)$ and $\delta=$ B-diam $(A)$. Then the family $\mathcal{T}$ has a common fixed point if and only if $\bigcap_{T \in \mathcal{T}} T(C)$ is not empty and $\mathcal{T}$-orbits are bounded.

Proof Clearly, if $\mathcal{T}$ has a fixed point, then we have $\bigcap_{T \in \mathcal{T}} T(C)$ is not empty and $\mathcal{T}$-orbits are bounded. So, let us assume that $\bigcap_{T \in \mathcal{T}} T(C)$ is not empty and $\mathcal{T}$-orbits are bounded. Let $x \in \bigcap_{T \in \mathcal{T}} T(C)$. The orbit $A=\mathcal{T}(x)$ is bounded. Note that $T(A)=A$ for any $A \in \mathcal{T}$. Indeed, by the definition of the orbit $\mathcal{T}(x)$, we have $T(A) \subset A$. Let $a \in A$, then there exists $S \in \mathcal{T}$ such that $a=S(x)$. Clearly, we have $a=T\left(T^{-1} \circ S(x)\right)$. Since $T^{-1} \circ S \in \mathcal{T}$, we conclude that $a \in T(A)$. Next we consider the admissible subset $C=\bigcap_{a \in A} \bar{B}(a, \delta)$, where $\delta=\mathrm{B}$-diam $(A)$. Obviously, $A \subset C$ and $C$ is a bounded and 1-local Bregman retract of $C$. As in the proof of the lemma above, one will easily show that $T(C) \subset C$ for any $T \in \mathcal{T}$. So, from Theorem 4.3, we conclude that $\mathcal{T}$ has a common fixed point and its fixed point set $\operatorname{Fix}(\mathcal{T})$ is 1-local Bregman retract of $C$.

Competing interests

The authors declare that they have no competing interests.

Authors' contributions

All authors contributed equally and significantly in writing this article. All authors read and approved the final manuscript.

\section{Author details}

'Department of Mathematics, King Abdulaziz University, P.O. Box 80203, Jeddah, 21589, Saudi Arabia. ${ }^{2}$ Department of Mathematics, Yasouj University, Yasouj, 75918, Iran.

\section{Acknowledgements}

The authors would like to express their thanks to the referees for their helpful comments and suggestions. The first and third authors gratefully acknowledge the support from the King Abdulaziz University, Jeddah, Saudi Arabia during this research.

Received: 8 January 2013 Accepted: 15 April 2013 Published: 29 April 2013

\section{References}

1. Espínola, R, Lorenzo, P, Nicolae, A: Fixed points, selections and common fixed points for nonexpansive-type mappings. J. Math. Anal. Appl. 382, 503-515 (2011)

2. Hussain, N, Khamsi, MA, Kirk, WA: One-local retract and Banach operator pairs in metric spaces. Appl. Math. Comput. 218, 10072-10081 (2012)

3. Hussain, N, Khamsi, MA, Latif, A: Banach operator pairs and common fixed points in hyperconvex metric spaces. Nonlinear Anal. 74, 5956-5961 (2011)

4. Khamsi, MA: One-local retract and common fixed point for commuting mappings in metric spaces. Nonlinear Anal. 27, 1307-1313 (1996)

5. Aksoy, AG, Khamsi, MA: Nonstandard Methods in Fixed Point Theory. Springer, Berlin (1990)

6. De Marr, R: Common fixed points for commuting contraction mappings. Pac. J. Math. 13, 1139-1141 (1963)

7. Browder, FE: Nonexpansive nonlinear operators in a Banach space. Proc. Natl. Acad. Sci. USA 54, 1041-1054 (1965)

8. Khamsi, MA, Lin, M, Sine, R: On the fixed points of commuting nonexpansive maps in hyperconvex spaces. J. Math. Anal. Appl. 168, 372-380 (1992)

9. Espínola, R, Hussain, N: Common fixed points for multimaps in metric spaces. Fixed Point Theory Appl. 2010, Article ID 204981 (2010)

10. Hussain, N, Jungck, G, Khamsi, MA: Nonexpansive retracts and weak compatible pairs in metric spaces. Fixed Point Theory Appl. 2012, Article ID 100 (2012)

11. Hussain, N, Khamsi, MA, Latif, A: Banach operator pairs and common fixed points in modular function spaces. Fixed Point Theory Appl. 2011, Article ID 75 (2011)

12. Hussain, N: Asymptotically pseudo-contractions, Banach operator pairs and best simultaneous approximations. Fixed Point Theory Appl. 2011, Article ID 812813 (2011)

13. Takahashi, W: Nonlinear Functional Analysis, Fixed Point Theory and Its Applications. Yokohama Publishers, Yokohama (2000)

14. Takahashi, W: Convex Analysis and Approximation of Fixed Points. Yokohama Publishers, Yokahama (2000) 
15. Bregman, LM: The relation method of finding the common point of convex sets and its application to the solution of problems in convex programming. U.S.S.R. Comput. Math. Math. Phys. 7, 200-217 (1967)

16. Chen, J, Li, Z: Banach operator pair and common fixed points for nonexpansive maps. Nonlinear Anal. 74, 3086-3090 (2011)

17. Bauschke, HH, Combettes, PL: Construction of best Bregman approximations in reflexive Banach spaces. Proc. Am. Math. Soc. 131(12), 3757-3766 (2003)

18. Bauschke, HH, Borwein, JM, Combettes, PL: Essential smoothness, essential strict convexity, and Legendre functions in Banach spaces. Commun. Contemp. Math. 3, 615-647 (2001)

19. Bonnas, JF, Shapiro, A: Perturbation Analysis of Optimization Problems. Springer, New York (2000)

20. Bauschke, HH, Borwein, JM: Legendre functions and the method of random Bregman functions. J. Convex Anal. 4, 27-67 (1997)

21. Butnariu, $D, C e n s o r, Y, R e i c h, S$ : Iterative averaging of entropic projections for solving stochastic convex feasibility problems. Comput. Optim. Appl. 8, 21-39 (1997)

22. Bauschke, HH, Borwein, JM, Combettes, PL: Bregman monotone optimization algorithms. SIAM J. Control Optim. 42 , 596-636 (2003)

23. Borwein, MJ, Reich, S, Sabach, S: A characterization of Bregman firmly nonexpansive operators using a new monotonicity concept. J. Nonlinear Convex Anal. 12(1), 161-184 (2011)

24. Reich, S, Sabach, S: Existence and approximation of fixed points of Bregman firmly nonexpansive mappings in reflexive Banach spaces. In: Fixed-Point Algorithms for Inverse Problems in Science and Engineering, pp. 299-314. Springer, New York (2010)

25. Reich, S, Sabach, S: Two strong convergence theorems for Bregman strongly nonexpansive operators in reflexive Banach spaces. Nonlinear Anal. 73, 122-135 (2010)

26. Martín-Márquez, V, Reich, S, Sabach, S: Right Bregman nonexpansive operators in Banach spaces. Nonlinear Anal. 75, 5448-5465 (2012)

27. Uuanh, Y-Y, Jeng, J-C, Kuo, T-Y, Hong, C-C: Fixed point and weak convergence theorems for point-dependent $\lambda$-hybrid mappings in Banach spaces. Fixed Point Theory Appl. 2011, Article ID 105 (2011)

28. Künzi, H-PA, Otafudu, OO: $q$-hyperconvexity in quasipseudometric spaces and fixed point theorems. J. Funct. Spaces Appl. 2012, Article ID 765903 (2012). doi:10.1155/2012/765903

29. Butnariu, D, lusem, AN: Totally Convex Functions for Fixed Points Computation and Infinite Dimensional Optimization. Kluwer Academic, Dordrecht (2000)

30. Kohsaka, F, Takahashi, W: Proximal point algorithms with Bregman functions in Banach spaces. J. Nonlinear Convex Anal. 6(3), 505-523 (2005)

31. Censor, Y, Lent, A: An iterative row-action method for interval convex programming. J. Optim. Theory Appl. 34, 321-358 (1981)

32. Hussain, N: Common fixed points in best approximation for Banach operator pairs with Ćirić type I-contractions. J. Math. Anal. Appl. 338, 1351-1362 (2008)

33. Khan, AR, Akbar, F: Best simultaneous approximations, asymptotically nonexpansive mappings and variational inequalities in Banach spaces. J. Math. Anal. Appl. 354, 469-477 (2009)

34. Pathak, HK, Hussain, N: Common fixed points for Banach operator pairs with applications. Nonlinear Anal. 69 2788-2802 (2008)

35. Rockafellar, RT: Monotone operators and the proximal point algorithm. SIAM J. Control Optim. 14, 877-898 (1976)

36. Rockafellar, RT: On the maximality of sums of nonlinear monotone operators. Trans. Am. Math. Soc. 149, 75-88 (1970)

37. Rockafellar, RT: Characterization of subdifferentials of convex functions. Pac. J. Math. 17, 497-510 (1966)

38. Rockafellar, RT: On the maximal monotonicity of subdifferential mappings. Pac. J. Math. 33, 209-216 (1970)

39. Zălinescu, C: Convex Analysis in General Vector Spaces. World Scientific, River Edge (2002)

40. Naraghirad, E, Takahashi, W, Yao, J-C: Generalized retraction and fixed point theorems using Bregman functions in Banach spaces. J. Nonlinear Convex Anal. 13(1), 141-156 (2012)

41. Butnariu, D, Resmerita, E: Bregman distances, totally convex functions and a method for solving operator equations in Banach spaces. Abstr. Appl. Anal. 2006, Article ID 84919 (2006)

42. Chen, G, Teboulle, M: Convergence analysis of a proximal-like minimization algorithm using Bregman functions. SIAM J. Optim. 3, 538-543 (1993)

43. Takahashi, W, Wong, N-C, Yao, J-C: Fixed point theorems and convergence theorems for generalized nonspreading mappings in Banach spaces. J. Fixed Point Theory Appl. (2012). doi:10.1007/s11784-012-0074-3

44. Kirk, WA: The fixed point property and mappings which are eventually nonexpansive. In: Theory and Applications of Nonlinear Operators of Accretive and Monotone Type. Lecture Notes in Pure and Appl. Math., vol. 178, pp. 141-147. Dekker, New York (1996)

doi:10.1186/1687-1812-2013-113

Cite this article as: Hussain et al.: Existence of common fixed points using Bregman nonexpansive retracts and

Bregman functions in Banach spaces. Fixed Point Theory and Applications 2013 2013:113. 